FORSCHUNG - AUSBILDUNG - WEITERBILDUNG

Bericht Nr. 70

\title{
A RANDOM DISCRETE VELOCITY MODEL AND APPROXIMATION OF THE BOLTZMANN EOUATION
}

Reinhard Illner und Wolfgang Wagner

UNIVERSITÄT KAISERSLAUTERN

Fachbereich Mathematik

Arbeitsgruppe Technomathematik

Postfach 3049

W-6750 Kaiserslautern 


\title{
A RANDOM DISCRETE VELOCITY MODEL AND APPROXIMATION OF THE BOLTZMANN EQUATION
}

\author{
by \\ Reinhard Illner ${ }^{*}$ and Wolfgang Wagner \\ Fachbereich Mathematik, Universität Kaiserslautern, \\ Postfach 3049, 6750 Kaiserslautern, Germany.
}

\begin{abstract}
An approximation procedure for the Boltzmann equation based on random choices of collision pairs from a fixed velocity set and on discrete velocity models is designed. In a suitable limit, the procedure is shown to converge to the time-discretized and spatially homogeneous Boltzmann equation.
\end{abstract}

1. Introduction. We are concerned with the relationships between discrete velocity models and the Boltzmann equation on the one hand, and discrete velocity models and particle approximation of the Boltzmann equation on the other hand. The investigation done in this article was originally inspired by the fundamental problem of approximating the Boltzmann equation with discrete velocity models; the random discrete velocity model which we present here offers a very simple solution to this problem. In addition, it may offer an interesting alternative to solving the Boltzmann. equation with particle simulation techniques.

We mention that the model presented here is just one (the simplest) of numerous possible random discrete velocity models which are conceivable. We restrict our analysis to the present model because of its simplicity, because it contains all the essential ingredients of these new models and because we have a convergence proof for this model.

Discrete velocity models of the Boltzmann equation (which we will abbreviate as DVMs in the rest of this article) were originally introduced with the idea to arrive at simpler model equations. Indeed, for some fundamental questions like shock wave structures, boundary layers etc. the easiest models, like the Carleman model or the Broadwell model, offer analytic solutions (see Broadwell ${ }^{1}$ or Platkowski ${ }^{2}$ ). For more sophisticated models it does unfortunately not seem to be true that DVMs are simpler than the Boltzmann equation. In fact, the discretization of the velocity space prevents the application of the modern technique known as "velocity averaging", such that the famous global existence theorem for the Boltzmann equation due to DiPerna and Lions ${ }^{3}$ does not carry over to DVMs.

In spite of this, DVMs have found intensive attention over the decades, and this 
attention has led to impressive progress in kinetic theory and related fields (like, e.g., cellular automata). As for DVMs, there are global existence theorems for small data (Bony ${ }^{4}$ ), existence theorems for steady boundary value problems (Cercignani, Illner and Shinbrot ${ }^{5}$ ), explicit solutions (Cornille ${ }^{6}$ and Cabannes ${ }^{7}$ ) and numerical solutions (Greenberg ${ }^{8}$ ). Survey papers or books on discrete velocity models were published by Illner and Platkowski ${ }^{9}$ and by Sultangazin ${ }^{10}$. Recently, Nurlybaev ${ }^{11}$ has presented a systematic way to obtain DVMs from the Boltzmann equation.

It is remarkable that there seem to be no results on the approximation problem of the Boltzmann equation by DVMs. We believe that this is due to the closure problem for discrete velocity models, which is as follows:

If $\left(\xi_{1}, \ldots, \xi_{n}\right)$ is the admissible velocity set for a DVM, the collision rules for this DVM must be such that if $\left(\xi_{i}, \xi_{j}\right)$ is an admissible pair for a collision, then a possible outcome $\left(\xi_{i}^{\prime}, \xi_{j}^{\prime}\right)$ of this collision must result with velocities in the original set. For a general choice of original velocities, it is then not at all clear how many (if any) admissible pairs there are. Typically, some velocity pairs have to be excluded from collisions altogether, while others can have only very few types of collisions (i.e., only one or two outcomes of the collision are possible). In contrast, recall that in reality for any pre-collisional pair $\left(\xi, \xi_{m}\right)$ we have a continuum of post-collisional pairs $\left(\xi^{\prime}, \xi_{*}^{\prime}\right)$, given by

$$
\begin{aligned}
& \xi^{\prime}=\xi-e\left(e \cdot\left(\xi-\xi_{*}\right)\right) \\
& \xi_{*}^{t}=\xi_{*}+e\left(e \cdot\left(\xi-\xi_{*}\right)\right)
\end{aligned}
$$

$e \in S^{2}$. Recall that the spatially homogeneous Boltzmann equation is, in the usual notation $f_{*}=f\left(t, \xi_{*}\right), f^{\prime}=f\left(t, \xi^{\prime}\right), f_{*}^{\prime}=f\left(t, \xi_{*}^{\prime}\right)$

$$
\partial_{t} f=\iint q\left(\xi, \xi_{*}, e\right)\left\{f^{\prime} f_{*}^{\prime}-f f_{*}\right\} d e d \xi_{*}
$$

A weak formulation of the Boltzmann equation is obtained by multiplication of (1) with a continuous bounded test function $\varphi(\xi)$ and by integration over $\xi$ :

$$
\partial_{*} \iint \varphi d \xi=\iiint q\left(\xi_{,} \xi_{*}, \epsilon\right) \varphi(\xi)\left\{f^{\prime} f_{*}^{\prime}-f f_{*}\right\} d e d \xi_{*} d \xi
$$

We write $\mu_{t}$ for the measure with density $f(t, \xi)$, then $(2)$ can be rewritten as

$$
\partial_{t}\left\langle\mu_{t}, \varphi\right\rangle=\iiint q\left(\xi, \xi_{*}, e\right) \varphi(\xi)\left(d\left(M_{t} \circ J\right)\left(\xi, \xi_{*}, e\right)-d M_{t}\left(\xi, \xi_{*}, e\right)\right)
$$

where $J:\left(\xi, \xi_{*}, e\right) \rightarrow\left(\xi^{\prime}, \xi_{*}^{\prime},-e\right)$ denotes the involutive collision transtormation and $d M_{t}$ is the product measure

$$
d M_{i}\left(\xi, \xi_{*}, e\right)=d \mu_{i}(\xi) d \mu_{t}\left(\xi_{*}\right) d e
$$

DVMs approximating the Boltzmann equation must therefore be designed such that the totality of their loss part of the collision term approximates the product 
measure $d M_{t}$ and such that the totality of their gain parts approximates the measure $d\left(M_{t} \circ J\right)$.

The closure problem addressed above leads to severe difficulties at this very point. Notice that it is not enough to just approximate $d \mu_{t} \otimes d \mu_{t}$, we have to approximate $d \mu_{t}(\xi) d \mu_{t}\left(\xi_{*}\right)$ de ; however, since in the standard theory of DVMs some velocity pairs are not suitable for collisions at all, or only with very few collision parameters $e$, the approximation of the measure de, and hence the approximation of $d M_{t}$, becomes problematical.

We made some attempts to overcome the difficulty for standard DVMs, but without success. The models which we constructed towards this end may be of independent interest and will be discussed elsewhere, but we eventually overcame the approximation problem by the introduction of a new type of discrete velocity model, the random discrete velocity model (RDVM).

Random discrete velocity models are a hybrid between standard discrete velocity models and particle simulation techniques for the Boltzmann equation. The only fundamental idea is to work with a finite set of velocities at any given time, but to allow that set and/or the collision rules to change stochastically from time to time. Needless to say, this idea can be implemented in a multitude of ways. In this paper, we confine ourselves to the presentation of the simplest RDVM we were able to construct - at any time, the model consists of finitely many independent 4 -velocity models. We design the model in section 2. In section 3 , we prove convergence to the time discretized and spatially homogeneous Boltzmann equation

$$
f(t+\Delta t, \xi)=f(\xi, t)+\Delta t \iint d e d \xi_{*} q\left(\xi, \xi_{*}, e\right)\left\{f^{\prime} f_{*}^{\prime}-f f_{*}\right\}
$$

We require a special type of approximation of the initial value- we have to distinguish between "primary" (pre-collisional) and "secondary" (post-collisional) velocities, and only the primary velocities can be used to approximate the data. This is a subtlety of the proof which surprised us ourselves, but if the secondary velocities are allowed for the approximation of the intial data, the approximation of the collision term may be in jeopardy. The reason for this is explained in section 4.

We believe that random discrete velocity models as introduced here can be combined with the standard trick of partitioning the physical space into cells, spatial homogenization over each cell and "splitting" of free flow and collision calculation to prove convergence of the method to the full, spatially inhomogeneous Boltzmann equation. The blueprint for this procedure is contained in ref. 12 (Babovsky, Inner), and we relegate the detailed discussion to future work.

\section{A Random Discrete Velocity Model.}

2.1. The model. Let $\Re^{3}$ be the velocity space, and let $n=2 m$ be an even integer. Suppose that $\Re^{3}$ is partitioned into $n$ velocity cells; of course, some of these cells must have infinite volume. 
We will assume that as $n \rightarrow \infty$, the "mesh" of our partition converges to zero. More precisely, let for given $n C_{n}(j)$ be the $j$-th cell in the partition chosen for $n$, and let $B_{R}(0)=\{\xi ;\|\xi\| \leq R\}$. We require that

$$
\lim _{n \rightarrow \infty} \sup _{0 \leq j \leq n} \text { diameter }\left(C_{n}(j) \cap B_{R}(0)\right)=0
$$

for each $R>0$, i.e. the mesh of the partition converges to 0 uniformly on compact sets.

Of course, the refinement of the partition is needed to approximate the initial value and the solution of the Boltzmann equation by point measures.

We choose velocities $\xi_{i}\left(=\xi_{i}^{(n)}\right)$ such that for each $i=1, \ldots, n \xi_{i} \in C_{n}(i)$.

The following five steps define an RDVM based on an initial partition of $\Re^{3}$ into $n=2 m$ velocity cells and velocities $\xi_{i}, i=1, \ldots, n$, chosen from the cells.

1. Initialization. If $f_{0}(\xi) \in L_{+}^{1}$ is the initial value for the spatially homogeneous Boltzmann equation, we associate the initial weight $g_{i}(0)=\int_{C_{\mathrm{n}}(\mathrm{i})} f_{0}(\xi) d \xi$ with the velocity $\xi_{i}$. Note the obvious consequences

$$
g_{i}(0) \leq \sum_{i=1}^{n} g_{i}(0)=\int f_{0}=1,
$$

and

$$
\sum_{i, j} g_{i}(0) g_{j}(0)=\left(\sum_{i} g_{i}(0)\right)^{2}=1
$$

Replace $f_{0}(\xi) d \xi$ by $\sum_{i=1}^{n} g_{i}(0) \delta_{\xi_{i}}(d \xi)$. We denote this discrete measure by $\mu_{0}^{n_{0}}$.

2. Collision rule. Choose randomly $m$ disjoint collision pairs of velocities $\left(\xi_{i}, \xi_{j}\right)$ from $\left\{\xi_{1}, \ldots, \xi_{n}\right\}$. This can be realized, conceptually, by choosing randomly a permutation $\pi$ of $\{1, \ldots, n\}$ and by forming the pairs $\left(\xi_{\pi(2 k-1)}, \xi_{\pi(2 k)}\right), k=1, \ldots, m$. Then, choose $m$ independent and equidistributed random collision parameters $e_{k} \in S^{2}$ and calculate post-collisional (secondary) velocities $\left(\xi_{\pi(2 k-1)}^{\prime}, \xi_{m(2 k)}^{\prime}\right)$ according to the col lision transformation

$$
\begin{aligned}
\xi_{\pi(2 k-1)} & =\xi_{\pi(2 k-1)}-e_{k}\left(e_{k} \cdot\left(\xi_{\pi(2 k-1)}-\xi_{\pi(2 k)}\right)\right) \\
\xi_{\pi(2 k)}^{\prime} & =\xi_{\pi(2 k)}+e_{k}\left(e_{k} \cdot\left(\xi_{\pi(2 k-1)}-\xi_{\pi(2 k)}\right)\right)
\end{aligned}
$$

This way of choosing random collision pairs was first suggested by Babovsky, see ref. 13.

3. Time evolution. Let $\Delta t>0$ be a time step. We calculate the mass transfer from the pre-collisional to the post-collisional velocities according to the formulas

$$
\begin{aligned}
& g\left(0, \xi_{i}\right)=g_{i}(0), i=1, \ldots, n \\
& g\left(0, \xi_{i}^{\prime}\right)=0, i=1, \ldots, n
\end{aligned}
$$


(notice that we take zero initial data for the post-collisional velocities, as mentioned in the introduction), and

$$
\begin{gathered}
\tilde{g}\left(\Delta t, \xi_{i}\right)=g\left(0, \xi_{i}\right)-F(i, j, e) \\
\tilde{g}\left(\Delta t, \xi_{j}\right)=g\left(0, \xi_{j}\right)-F(j, i, e) \\
\tilde{g}\left(\Delta t, \xi_{i}^{\prime}\right)=F(i, j, e) \\
\tilde{g}\left(\Delta t, \xi_{j}^{\prime}\right)=F(j, i, e)
\end{gathered}
$$

where $\left(\xi_{i}, \xi_{j}\right)$ forms, of course, one of the chosen collision pairs, $e$ is the corresponding collision parameter, and

$$
F(i, j, e):=\left\{\begin{array}{l}
\text { a) } \Delta t \cdot 2 m \cdot\left|s^{2}\right| q\left(\xi_{i}, \xi_{j}, e\right) g\left(0, \xi_{j}\right) g\left(0, \xi_{i}\right) \\
\text { if this quantity is } \leq g\left(0, \xi_{i}\right) \\
\text { b) } g\left(0, \xi_{i}\right) \text { otherwise. }
\end{array}\right.
$$

$\left|S^{2}\right|=4 \pi$ is the surface of the unit sphere.

Notice that a) in the definition of $F(i, j, e)$ applies if $\Delta t$ is so small that

$$
\Delta t(2 m)\left|s^{2}\right| q\left(\xi_{i}, \xi_{j}, e\right) g\left(0, \xi_{j}\right)<1
$$

for all $i, j$. Condition (7) is reasonable at time zero because we expect from the definition of the weights $g\left(0, \xi_{j}\right)$ that

$$
g\left(0, \xi_{j}\right) \leq \frac{C}{2 m}
$$

for some constant $C$. Whether (8) will remain true in the time evolution is an important but difficult question which we leave for future work. Obviously, the constraint (7) on $\Delta t$ becomes problematical as the number of velocities (and hence weights) increases.

The result of our time evolution is a discrete measure

$$
\tilde{\mu}_{\Delta t}^{n}=\sum_{i=1}^{n} \tilde{g}_{i}(\Delta t) \delta_{\xi_{i}}+\sum_{i=1}^{n} \ddot{g}_{i}^{\prime}(\Delta t) \delta_{\xi_{i}}
$$

where $\ddot{g}_{i}(\Delta t)=\ddot{g}\left(\Delta t, \xi_{i}\right)$ and $\ddot{g}_{i}^{\prime}(\Delta t)=\ddot{g}\left(\Delta t, \xi_{i}^{\prime}\right)$.

4. Redistribution of the weights associated with the post-collisional veloc-. ities. Consider a cell $C_{n}(i)$. It carries now the weight $\tilde{g}_{3}(\Delta t)$ plus possible weights $g\left(\Delta l, \xi_{i_{k}}^{\prime}\right), k=1, \ldots, L$, whose (post-collisional) velocities $\xi_{i_{1}}^{\prime}, \ldots, \xi_{i_{L}}^{\prime}$ fell in $C_{n}(i)$, In this case, we set

$$
g_{i}(\Delta t)=g\left(\Delta t, \xi_{i}\right)=\ddot{g}\left(\Delta t, \xi_{i}\right)+\sum_{k=1}^{t} g\left(\Delta t, \xi_{i_{k}}\right)
$$

and $\mu_{\Delta t}^{n}=\sum_{i=1}^{n} g_{i}(\Delta t) \delta_{s ;}$ 
5. Iteration. Return to step 2: Choose new random pairs, new random collision parameters, etc.

As simple as this procedure is, we will prove below that it converges in a suitable

limit to the Boltzmann equation. It is for this convergence proof that we had to choose the normalizing constants $2 m$ and $\left|S^{2}\right|=4 \pi$ in (6).

2.2. The connection with discrete velocity models. The definition (5) arises, of course, from the roughest possible (Euler) discretization of the spatially homogeneous discrete velocity model

$$
\begin{aligned}
& \frac{d}{d t} g\left(t, \xi_{i}\right)=(2 m)\left|S^{2}\right| q\left(\xi_{i}, \xi_{j}, e\right)\left(g\left(t, \xi_{i}^{\prime}\right) g\left(t, \xi_{j}^{\prime}\right)-g\left(t, \xi_{j}\right) g\left(t, \xi_{j}\right)\right) \\
& \frac{d}{d t} g\left(t, \xi_{j}\right)=(2 m)\left|S^{2}\right| q\left(\xi_{i}, \xi_{j}, e\right)\left(g\left(t, \xi_{i}^{\prime}\right) g\left(t, \xi_{j}^{\prime}\right)-g\left(t, \xi_{i}\right) g\left(t, \xi_{j}\right)\right) \\
& \frac{d}{d t} g\left(t, \xi_{i}^{\prime}\right)=-(2 m)\left|S^{2}\right| q\left(\xi_{i}, \xi_{j}, e\right)\left(g\left(t, \xi_{i}^{\prime}\right) g\left(t, \xi_{j}^{\prime}\right)-g\left(t, \xi_{i}\right) g\left(t, \xi_{j}\right)\right) \\
& \frac{d}{d t} g\left(t, \xi_{j}^{\prime}\right)=-(2 m)\left|S^{2}\right| q\left(\xi_{i}, \xi_{j}, e\right)\left(g\left(t, \xi_{i}^{\prime}\right) g\left(t, \xi_{j}^{\prime}\right)-g\left(t, \xi_{i}\right) g\left(t, \xi_{j}\right)\right)
\end{aligned}
$$

where we have modified the scheme such that the $g$ are assured to be nonnegative and chosen the initial values associated with secondary velocities (to be more precise, secondary configurations $\left.\left(\xi_{\pi(2 k-1)}^{\prime}, \xi_{\pi(2 k)}^{\prime}, c_{k}\right)\right)$, as zero. This turns out not to be just: a matter of convenience. Our convergence proof, as we will explain later, works only for this particular choice.

It is of course well known that (11) with such data has a nonnegative global solution. We approximate this solution on $(0, \Delta t)$ in the simplest possible way. Therefore, the scheme described in steps $1-5$ is based on an Euler discretization of $\mathrm{m}$ 4-velocity (Broadwell type) models, with a specific choice for the initial values. After each time step the collision rules are changed, and a new model emerges, for which the initial data must be adapted.

\section{A Convergence Theorem.}

\subsection{Preparations.}

Let $t \in\{k \cdot \Delta t ; k=0,1,2, \ldots\}$. Consider the time discretized spatially homogeneous Boltzmann equation

$$
f(t+\Delta t, \xi)=f(\xi, t)+\Delta t \iint d e d \xi_{*} q\left(\xi, \xi_{*}, e\right)\left\{f^{\prime} f_{*}^{\prime} \cdots f f_{*}\right\}
$$

Let $C_{b}\left(\Re^{3}\right)$ be the space of all continuous bounded functions. After multiplication with a test function $\varphi \in C_{b}\left(\Re^{3}\right)$, integration and the usual application of the collision transformation and symmetry properties we find

$$
\left\langle\mu_{t+\Delta t} \varphi\right\rangle=\left\langle\mu_{t}, \varphi\right\rangle+\Delta t \iiint q\left(\xi, \xi_{*}, c\right) \frac{1}{2}\left\{\varphi^{\prime}+\varphi_{*}^{\prime}-\varphi-\varphi_{*}\right\} d \mu_{t}(\xi) d \mu_{t}\left(\xi_{*}\right) d c
$$


where $d \mu_{t}(\xi)=f(t, \xi) d \xi$. As for the collision kernel $q$, we assume that it is invariant under the collision transformation and otherwise a bounded and continuous function of its arguments. The second and third assumption, of course, require a truncation in all cases of practical interest; for example, in the hard sphere case we have to replace $\left|\left(\xi-\xi_{*}\right) \cdot e\right|$ for large relative velocities by something bounded, and such that the result is continuous.

Our assumptions on the collision kernel $q$ imply that $\Delta t$ can be chosen so small that $\mu_{t+\Delta t}$ is a probability measure if $\mu_{t}$ is one. Specifically, we have to choose $\Delta t$ such that

$$
\Delta t \cdot \int q d e \leq 1
$$

to assure positivity of $\mu_{t+\Delta t}$. There is in fact no need here to confine the discussion to absolutely continuous measures, because (12) is well defined for general probability measures $\mu_{t}$ and does in fact define $\mu_{t}$ for all $t \in \mathbb{N}$ if $\mu_{0}$ is given. Equation (12) is our targetted limit equation.

We abbreviate $g\left(t, \xi_{i}\right)$ and $g\left(t, \xi_{i}\right)$ by $g_{i}(t)$ and $\ddot{g}_{i}(t)$ respectively and denote by $\mu_{i}^{n}$, $\ddot{\mu}_{t}^{n}$ the random point measures generated iteratively by the procedure from section 2. Specifically,

$$
\begin{aligned}
\mu_{0}^{n} & =\sum_{i=1}^{n} g_{i}(0) \delta_{\xi_{i}}, \\
\mu_{\Delta t}^{n} & =\sum_{i=1}^{n} g_{i}(\Delta t) \delta_{\xi_{i}}+\sum_{i=1}^{n} g_{i}^{\prime}(\Delta t) \delta_{\xi_{i}^{\prime},} \\
\mu_{\Delta t}^{n} & =\sum_{i=1}^{n} g_{i}(\Delta t) \delta_{\xi_{i}} .
\end{aligned}
$$

Our objective is a comparison of $\mu_{k \Delta t}^{n}$ with $\mu_{k \Delta t}$, where $\mu_{0}(\xi)=f_{0}(\xi) d \xi$, and $\mu_{(k+1) \Delta t}$ is given by (12).

We will exploit the equivalence between weak-* convergence of bounded measures and convergence in the bounded Lipschitz distance. For simplicity, we only consider probability measures on $\Re^{3}$. The bounded Lipschitz distance between two probability measures $\mu$ and $\nu$ is defined by

$$
\rho(\nu, \mu)=\sup _{\varphi \in D}\left|\int \varphi d \mu-\int \varphi d \nu\right|
$$

where

$$
D=\left\{\varphi: \Re^{3} \rightarrow[0,1] ;|\varphi(x)-\varphi(y)| \leq\|x-y\|\right\}
$$

Lemma 3.1. (see ${ }^{14}$, Corollary 3.5) Let $\left\{\mu^{n}\right\}$ be a sequence of random probability measures and let $\mu$ be a probability measure on $\Re^{3}$. Then the following are equivalent:

a) $\forall c>0 \operatorname{Prob}\left\{\rho\left(\mu^{n} ; \mu\right)>\epsilon\right\} \rightarrow 0$ as $n \rightarrow \infty$ 
b) $\forall \epsilon>0 \forall \varphi \in C_{b}\left(\Re^{3}\right) \operatorname{Prob}\left\{\left|\left\langle\mu^{n}, \varphi\right\rangle-\langle\mu, \varphi\rangle\right|>\epsilon\right\} \rightarrow 0$ as $n \rightarrow \infty$

c) $\forall \varphi \in C_{b}\left(\Re^{3}\right) \lim _{n \rightarrow \infty} E\left\langle\mu^{n}, \varphi\right\rangle=\langle\mu, \varphi\rangle$

and $\lim _{n \rightarrow \infty} \operatorname{var}\left\langle\mu^{n}, \varphi\right\rangle=0$.

Lemma 3.2. Suppose that $\ddot{\nu}_{n}$ is a sequence of random probability measures such that

$$
\tilde{\nu}_{n} \rightarrow \nu
$$

in probability, and define

$$
\nu_{n}(d \xi)=\sum_{i} \tilde{\nu}_{n}\left(C_{n}(i)\right) \delta_{\xi_{i}}(d \xi)
$$

If (4) holds for each $R>0$, then in probability

$$
v_{n} \rightarrow \nu
$$

as $n+\infty$.

Proof. For $\varphi \in D$, we have

$$
\begin{aligned}
\left|\left\langle\tilde{\nu}_{n}, \varphi\right\rangle-\left\langle\nu_{n}, \varphi\right\rangle\right| & =\mid \sum\left[\tilde{\nu}_{n}\left(C_{n}(i)\right) \varphi\left(\xi_{i}\right)-\int_{C_{n}(i)} \varphi(\xi) d \tilde{\nu}_{n}(\xi)||\right. \\
& \left.=\left|\sum_{i} \int_{C_{n}(i)}\right| \varphi\left(\xi_{i}\right)-\varphi(\xi)\right] d \tilde{\nu}_{n}(\xi) \mid \\
& \leq \sum_{i} \int_{C_{n}(i) n B_{n}}\left|\varphi\left(\xi_{i}\right)-\varphi(\xi)\right| d \tilde{\nu}_{n}(\xi)+2\|\varphi\|_{L \infty} \tilde{\nu}_{n}\left(B_{R}^{c}\right)
\end{aligned}
$$

Therefore,

$$
\begin{aligned}
\rho\left(\nu_{n}, \check{\nu}_{n}\right) & \leq \sum_{i} \operatorname{diameter}\left(C_{n}(i) \cap B_{R}\right) \tilde{\nu}_{n}\left(C_{n}(i) \cap B_{R}\right)+2 \tilde{\nu}_{n}\left(B_{R}^{c}\right) \\
& \leq \sup _{i} \text { diameter }\left(C_{n}(i) \cap B_{R}\right)+2 \ddot{\nu}_{n}\left(B_{R}^{c}\right)
\end{aligned}
$$

and for all $R>0$

$$
\lim _{n \rightarrow \infty} E p\left(\nu_{n_{1}}, \tilde{\nu}_{n}\right) \leq 2 \lim _{n \rightarrow \infty} E \tilde{\nu}_{n}\left(B_{R}^{c}\right)=2 E \nu\left(B_{R}^{c}\right)
$$

As $E v$ defines a probability measure, $\lim _{n \rightarrow \infty} E \nu=0$. It follows that $\lim _{n \rightarrow \infty} E \rho\left(\tilde{\nu}_{n}, \nu_{n}\right)=$ 0 , and the triangle incquality

$$
\rho\left(v_{n}, \nu\right) \leq \rho\left(\nu_{n}, \ddot{\nu}_{n}\right)+\rho\left(\ddot{\nu}_{n}, \nu\right)
$$

implies the assertion. 
Corollary $\mathbf{3 . 3 .}$

$$
\lim _{n \rightarrow \infty} \sum_{i=1}^{n} g_{i}(0) \delta_{\xi_{i}}(d \xi)=f_{0}(\xi) d \xi
$$

weak - * in the sense of bounded measures.

Proof. This follows from Lemma 3.2, the definition of the weights $g_{i}(0)$ and the property (4) of the cell partition.

\subsection{The main Theorem}

The following Theorem contains the main step towards convergence of our scheme.

Theorem 3.4. Suppose that we already know that in probability

$$
\mu_{t}^{n} \rightarrow \mu_{t} \text { as } n \rightarrow \infty
$$

assume that condition $(4)$ is satisfied, and assume that the stochastic weights $g_{i}(t)$ obtained from the scheme in section 2 satisfy the conditions

$$
A .1 \exists C>0 \text { such that } \operatorname{Prob}\left\{n \cdot \sup _{i} g_{i}(t)>C\right\} \rightarrow 0 \text { as } n \rightarrow \infty
$$

and

$$
A .2 \sup _{n} E\left(n \cdot \sup _{i} g_{i}(t)\right)^{2}<\infty
$$

If then $\Delta t \leq\left(8 \pi C q_{\max }\right)^{-1}$, we have

$$
\mu_{t+\Delta t}^{n} \rightarrow \mu_{t+\Delta t}
$$

in probability (in any of the three equivalent forms spelled out in Lemma 3.1). The constant $C$ is the one from $A, 1$, and $q_{\max }$ is a shorthand for the lowest upper bound of $q$.

Remarks. The conditions $A .1$ and $A .2$ mean that we assume that the normalization $g_{i}(t) \leq C / n$, which is expected for $t=0$, remains with large probability valid uniformly in $\mathrm{n}$ for all $t=k \Delta t, k=1,2, \ldots$ This corresponds to $t^{\infty}$-control uniformly in $n$ for the discrete velocity model. We strongly believe that this is a realistic assumption, at least if the initial value for the Boltzmann equation is in $L^{\infty}$, but have presently no proof for it. An investigation of the validity of $A .1$ and A.2 is planned for future work.

Proof of Theorem 3.4. In view of Lemma 3.2, it is enough to show that $\mu_{i+\Delta t}^{n} \rightarrow \mu_{t+\Delta t}$ in probability.

Choose a $\varphi \in C_{b}\left(\Re^{3}\right)$. With this choice made, we introduce the following abbre viations:

$$
\begin{gathered}
G(i, j, e):=\left[\varphi\left(\xi_{i}\right)-\varphi\left(\xi_{i}\right)\right] F(i, j, e)+\left[\varphi\left(\xi_{j}^{\prime}\right)-\varphi(\xi,)\right] F(j, i, e) \\
A(i, j):=\frac{1}{4 \pi} \int_{S^{2}} G(i, j, e) d e
\end{gathered}
$$




$$
B(i, j):=\frac{1}{4 \pi} \int_{S^{2}}[G(i, j, e)]^{2} d e
$$

Then

$$
\begin{aligned}
\left\langle\tilde{\mu}_{t+\Delta t}^{n}, \varphi\right\rangle= & \sum_{k=1}^{m}\left\{\varphi\left(\xi_{*(2 k-1)}\right) \dot{g}_{\pi(2 k-1)}(t+\Delta t)+\varphi\left(\xi_{\pi(2 k)}\right) \tilde{g}_{\pi(2 k)}(t+\Delta t)\right. \\
& \left.+\varphi\left(\xi_{\pi(2 k-1)}^{\prime}\right) \dot{g}_{\pi(2 k-1)}^{\prime}(t+\Delta t)+\varphi\left(\xi_{\pi(2 k)}^{\prime}\right) \dot{g}_{\pi(2 k)}^{\prime}(t+\Delta t)\right\} \\
= & \sum_{k=1}^{m}\left\{\varphi\left(\xi_{\pi(2 k-1)}\right) g_{\pi(2 k-1)}(t)+\varphi\left(\xi_{\pi(2 k)}\right) g_{\pi(2 k)}(t)\right\} \\
+ & \sum_{k=1}^{m}\left\{\left[\varphi\left(\xi_{\pi(2 k-1)}^{\prime}\right)-\varphi\left(\xi_{\pi(2 k-1)}\right)\right] F\left(\pi(2 k-1), \pi(2 k), e_{k}\right)\right. \\
& \left.+\left[\varphi\left(\xi_{\pi(2 k)}^{\prime}\right)-\varphi\left(\xi_{\pi(2 k)}\right)\right] F\left(\pi(2 k), \pi(2 k-1), e_{k}\right)\right\}
\end{aligned}
$$

or briefly

$$
\left\langle\tilde{\mu}_{t+\Delta t}^{n}, \varphi\right\rangle=\left\langle\mu_{t}^{n}, \varphi\right\rangle+\sum_{k=1}^{m} G\left(\pi(2 k-1), \pi(2 k), e_{k}\right\rangle
$$

By assumption, the first term on the right converges in probability to $\left\langle\mu_{t}, \varphi\right\rangle$. We calculate the limit of the sum by computing the firs? two moments (i.e. expectation and variance) and by applying Lemma 3.1. To this end, let

$$
\eta=\sum_{k=1}^{m} G\left(\pi(2 k-1), \pi(2 k), e_{k}\right)
$$

We denote by $E\left(\eta \mid g_{i}(t)\right)$ the conditional expectation of $\eta$ given the random weights $g_{i}(t)$. By using the identity $E(\eta)=E\left(E\left(\eta \mid g_{i}(t)\right)\right)$ and because there are $(2 \mathrm{~m})$ ! permutations of $\{1, \ldots, 2 m\}$, we find

$$
\begin{aligned}
E\left(\eta \mid g_{i}(t)\right)=\sum_{\pi} \frac{1}{(2 m) !} \prod_{l=1}^{m} \frac{1}{4 \pi} \int \eta d e_{l}, \\
E(\eta)=E \sum_{\pi} \frac{1}{(2 m) !} \sum_{k=1}^{m} \frac{1}{4 \pi} \int_{S^{2}} G(\pi(2 k-1), \pi(2 k), e) d e \\
=E \sum_{\pi} \frac{1}{(2 m) !} \sum_{k=1}^{m} A(\pi(2 k-1), \pi(2 k)) .
\end{aligned}
$$

We rewrite this in terms of possible pairs $(i, j), i \neq j, i, j \in\{1, \ldots, 2 m\}$. Clearly, for every such pair there are $(2 m-2) ! \cdot m$ permutations $\pi$ such that $\pi(2 k-1)=i$, 
$\pi(2 k)=j$ ( $m$ possibilities to place the pair $(i, j)$, times the $(2 \mathrm{~m}-2) !$ possibilities to permute the remaining indices). Therefore,

$$
\begin{aligned}
& E(\eta)=E \frac{1}{(2 m) !} \sum_{i \neq j} A(i, j) \cdot(2 m-2) ! m \\
& =\quad E_{\frac{1}{2(2 m-1)}} \sum_{i, j} A(i, j) .
\end{aligned}
$$

In the last step we have used that for $i=j, \xi_{i}^{\prime}=\xi_{i}$ and $\xi_{j}^{\prime}=\xi_{j}$ and thus $A(i, i)=0$ by definition.

Before we continue the evaluation of $E(\eta)$, we use similar arguments for a reduction of $E\left(\eta^{2}\right)$ :

$$
\begin{aligned}
E\left(\eta^{2}\right)= & E \sum_{\pi} \frac{1}{(2 m) !} \prod_{l=1}^{m} \frac{1}{4 \pi} \int_{S^{2}} d e_{l}\left(\sum_{k=1}^{m} G\left(\pi(2 k-1), \pi(2 k), e_{k}\right)\right)^{2} \\
= & E \sum_{\pi} \frac{1}{(2 m) !} \prod_{i=1}^{m} \frac{1}{4 \pi} \int_{S^{2}} d e_{l}\left\{\sum_{k=1}^{m} G\left(\pi(2 k-1), \pi(2 k), e_{k}\right)^{2}\right. \\
+ & \left.\sum_{k \neq m} G\left(\pi(2 k-1), \pi(2 k), e_{k}\right) \cdot G\left(\pi(2 r-1), \pi(2 r), e_{r}\right)\right\} \\
= & E \sum_{\pi} \frac{1}{(2 m) !} \sum_{k=1}^{m} \frac{1}{4 \pi} \int_{S^{2}} d e\left(G(\pi(2 k-1), \pi(2 k), e)^{2}\right. \\
& +E \sum_{\pi} \frac{1}{(2 m) !} \sum_{k \neq r} A(\pi(2 k-1), \pi(2 k)) A(\pi(2 r-1), \pi(2 r)) \\
= & E \frac{1}{(2 m) !}(2 m-2) ! m \sum_{i, j} B(i, j) \\
& +E \frac{1}{(2 m) !} \sum_{i, j, \alpha, \beta}^{*}(2 m-4) ! m(m-1) A(i, j) A(\alpha, \beta) .
\end{aligned}
$$

The symbol $\sum^{*}$ means that the sum is over all indices $i, j, \alpha, \beta$ for which no two of these are equal. Nlso, we have used that for fixed pairs $i \neq j$ and $\alpha \neq \beta$, there are $(2 \mathrm{~m}-4) ! \mathrm{m}(\mathrm{m}-1)$ permutations $\pi$ such that there are $k$ and $r, k \neq r$ with $\pi(2 k-1)=i, \pi(2 k)=j, \pi(2 r-1)=\alpha, \pi(2 r)=\beta$. The last expression can be rewritten as

$$
\begin{gathered}
E\left(\eta^{2}\right)=E \frac{1}{2(2 m-1)} \sum_{i, j} B(i, j)+E_{\frac{1}{4(2 m-1)(2 m-3)}\left(\sum_{i, j} A(i, j)\right)^{2}} \\
-E_{\frac{1(2 m-1)(2 m-3)}{2}} \sum_{i, j, \alpha, \beta}^{* *} A(i, j) A(\alpha, \beta),
\end{gathered}
$$

where $\sum^{* * *}$ means that at least two of the indices $i, j, \alpha, \beta$ are equal. Notice that the second term on the right of (17) is equal to

$$
E\left(\frac{1}{2(2 m-1)} \sum_{i, j} A(i, j)\right)^{2} \cdot \frac{2 m-1}{2 m-3}
$$


The next step is the investigation of the various terms, where we use, for the first time, the specific form of the functions $F(i, j, e)$. Let

$$
F_{1}(i, j, e):=8 \pi \Delta t m q\left(\xi_{i}, \xi_{j}, e\right) g_{i}(t) g_{j}(t)
$$

and

$$
I(i, j, e)=\left\{\begin{array}{l}
1, F_{1}(i, j, e)>g_{i}(t) \\
0, \text { otherwise }
\end{array}\right.
$$

Clearly, then $F(i, j, e)=F_{1}(i, j, e)[1-I(i, j, e)]+g_{i}(t) I(i, j, e)$, and

$$
\begin{aligned}
\frac{1}{2(2 m-1)} \sum_{i, j} A(i, j)= & \frac{1}{2(2 m-1)} \sum_{i, j} \frac{1}{4 \pi} \int d e\left\{\left[\varphi\left(\xi_{i}\right)-\varphi\left(\xi_{i}\right)\right] F(i, j, e)\right. \\
& \left.+\left[\varphi\left(\xi_{j}^{\prime}\right)-\varphi\left(\xi_{j}\right)\right] F(j, i, e)\right\} \\
= & \frac{1}{2 m-1} \sum_{i, j} \frac{1}{4 \pi} \int \operatorname{de}\left[\varphi\left(\xi_{i}\right)-\varphi\left(\xi_{i}\right)\right] F(i, j, e) \\
= & \frac{1}{2 m-1} \sum_{i, j} \frac{1}{4 \pi} \int d e\left[\varphi\left(\xi_{i}\right)-\varphi\left(\xi_{i}\right)\right] F_{1}(i, j, e) \\
& +\frac{1}{2 m-1} \sum_{i, j} \frac{1}{4 \pi} \int d e\left[\varphi\left(\xi_{i}\right)-\varphi\left(\xi_{i}\right)\right]\left[g_{i}(t)-F_{1}(i, j, e)\right] I(i, j, e) \\
= & \frac{2 m}{2 m-1} \Delta t \sum_{i, j} \int d e\left[\varphi\left(\xi_{i}\right)-\varphi\left(\xi_{i}\right)\right] q\left(\xi_{i}, \xi_{j}, e\right) g_{i}(t) g_{j}(t) \\
& +R_{1} \cdot
\end{aligned}
$$

From the definition of $\mu_{t}^{n}$, we find

$\frac{1}{2(2 m-1)} \sum_{i, j} A(i, j)=R_{1}+\frac{2 m}{2 m-1} \Delta t \iiint d e\left[\varphi\left(\xi^{\prime}\right)-\varphi(\xi)\right] q\left(\xi, \xi_{*}, e\right) d \mu_{t}^{n}(\xi) d \mu_{t}^{n}\left(\xi_{*}\right)$

As by definition

$$
g_{i}(t) I(i, j, e) \leq F_{1}(i, j, e) I(i, j, e)
$$

we can estimate

$$
\begin{aligned}
\left|R_{1}\right| & \leq \frac{1}{2 m-1} 2\|\varphi\|_{L^{\infty}} \sum_{i, j} \frac{1}{4 \pi} \int \operatorname{de} F_{1}(i, j, e) I(i, j, e) \\
& \leq \text { const. } \sum_{i, j} g_{i}(t) g_{j}(t) \Pi(i, j, e) .
\end{aligned}
$$

Now let

$$
I_{1}= \begin{cases}1, & \text { if } 8 \pi \Delta t m q_{\max } \max _{j} g_{j}(t)>1 \\ 0, & \text { otherwise. }\end{cases}
$$


Then by definition $I(i, j, e) \leq I_{1}$, and we obtain

$$
\left|R_{1}\right| \leq \text { const. } l_{1}
$$

Also, as $F(i, j, e) \leq F_{1}(i, j, e)$, we have the estimates

$$
\begin{gathered}
|G(i, j, e)| \leq 4\|\varphi\|_{L_{\infty}} F_{1}(i, j, e) \leq \text { const. } m g_{i}(t) g_{j}(t) \\
|A(i, j)| \\
|B(i, j)|
\end{gathered}
$$

As a consequence,

$$
\sum_{i, j}|A(i, j)| \leq \text { const. } m
$$

and

$$
\begin{aligned}
\sum_{i, j} B(i, j) & \leq \text { const. }\left\{m \sum_{i} g_{i}(t)^{2}\right\}^{2} \\
& \leq \text { const. }\left\{m \sup _{i} g_{i}(t)\right\}^{2} .
\end{aligned}
$$

Moreover, because $A(i, i)=0$ and $A(i, j)=A(j, i)$,

$$
\begin{aligned}
\sum_{i, j, \alpha, \beta}^{* *} A(i, j) A(\alpha, \beta) \leq & \operatorname{const.} \sum_{i, j, \beta} A(i, j) A(\alpha, \beta) \\
\leq & \text { const. } m^{2} \sum_{i, j, \beta} g_{i}(t)^{2} g_{j}(t) g_{\beta}(t) \\
& \leq \text { const. } m\left[m \sup _{i} g_{i}(t)\right] .
\end{aligned}
$$

Consider now the right hand side of (19). As $\mu_{t}^{n} \times \mu_{t}^{n} \rightarrow \mu_{t} \times \mu_{t}$ weak $-*$ in probability (or in probability in any of the equivalent ways spelled out in Lemma 3.1 ), and as the function

$$
\int_{S^{2}} d e\left[\varphi\left(\xi^{\prime}\right)-\varphi(\xi)\right] q\left(\xi, \xi_{*}, e\right)
$$

is continuous and bounded, the second expression in (19) converges in probability to

$$
T:=\Delta t \iiint_{\xi^{2}} d e\left[\varphi\left(\xi^{\prime}\right)-\varphi(\xi)\right] q\left(\xi_{,} \xi_{*}, e\right) d \mu_{t}(\xi) d \mu_{t}\left(\xi_{*}\right)
$$

The remaining term $R_{1}$ converges in probability to zero, because of (20) and by assumption A.1.

Summarizing, we have proved that in probability

$$
\frac{1}{2(2 m-1)} \sum_{i, j} A(i, j) \rightarrow T
$$

as $m \rightarrow \infty$. Furthermore, by $(22) \frac{1}{2(2 m-1)} \sum A(i, j)$ is bounded, and this implies that all moments also converge. In particular,

$$
E \frac{1}{2(2 m-1)} \sum_{i, j} A(i, j) \rightarrow T \text { as } m \rightarrow \infty
$$


and

$$
E\left\{\frac{1}{2(2 m-1)} \sum_{i, j} A(i, j)\right\}^{2} \rightarrow T^{2} \text { as } m \rightarrow \infty
$$

By (16) and (25) $E(\eta) \rightarrow T$ as $m \rightarrow \infty$, and by (17), (26), (23), (24) and assumption A.2 $E\left(\eta^{2}\right) \rightarrow T^{2}$ as $m \rightarrow \infty$. From Chebyshev's inequality we obtain that $\eta \rightarrow T$ in probability as $m \rightarrow \infty$, and from this and (15) we finally have that

$$
\left\langle\mu_{t+\Delta t}^{n}, \varphi\right\rangle \rightarrow\left\langle\mu_{t}, \varphi\right\rangle+T=\left\langle\mu_{t+\Delta t}, \varphi\right\rangle
$$

in probability as $\mathrm{n}(=2 m)$ converges to infinity.

\section{A remark.}

Recall that we set $g_{i}^{\prime}(0)=0, i=1, \ldots, n$, in section 2. This definition has the simple physical interpretation that post- collisional velocities need not be occupied at time 0 , because particles first have to experience collisions before they assume post-collisional velocities.

From a mathematical point of view, this reasoning is not quite satisfactory, because the distinction between pre- and post-collisional velocities is only an artifact of our model. For the full Boltzmann cquation, pro- and post-collisional velocities play fully symmetrical roles, due to the involutive property of the collision transformation.

The post-collisional velocities $\xi_{i}^{\prime}$ could also serve as auxiliary points to achieve a better point approximation of the initial measure $\mu_{0}$. Specifically, instead of approximating $\mu_{0}$ by $\sum_{i=1}^{n} g_{i}(0) \delta_{\xi_{i}}$, we could choose $g_{i}(0), g_{i}^{\prime}(0)$ such that

$$
\mu_{0}^{n}=\sum_{i=1}^{n_{n}} g_{i}(0) \delta_{\xi_{i}}+\sum_{i=1}^{n} g_{i}^{\prime}(0) \delta_{\xi_{i}^{\prime}}
$$

where at least some of the $g_{i}^{\prime}(0)$ would be positive.

Suppose that we choose this option. The time-discretized discrete velocity model we then have to study is

$$
\begin{aligned}
& \left.g_{i}(\Delta t)=g_{i}(0)+\Delta t(2 m)\left|S^{2}\right| q\left(\xi_{i}, \xi_{j}, e\right) \mid g_{i}^{\prime}(0) g_{j}^{\prime}(0)-g_{i}(0) g_{j}(0)\right] \\
& g_{j}(\Delta t)=g_{j}(0)+\Delta t(2 m)\left|S^{2}\right| q\left(\xi_{i}, \xi_{j}, e\right)\left[g_{i}^{\prime}(0) g_{j}^{\prime}(0)-g_{i}(0) g_{j}(0)\right] \\
& g_{i}^{\prime}(\Delta t)=g_{i}^{\prime}(0)-\Delta t(2 m)\left|S^{2}\right| q\left(\xi_{i}, \xi_{j}, e\right)\left[g_{i}^{\prime}(0) g_{j}^{\prime}(0)-g_{i}(0) g_{j}(0)\right] \\
& g_{j}^{\prime}(\Delta t)=g_{j}^{\prime}(0)-\Delta t(2 m)\left|S^{2}\right| q\left(\xi_{i}, \xi_{j}, e\right)\left[g_{i}^{\prime}(0) g_{j}^{\prime}(0)-g_{i}(0) g_{j}(0)\right]
\end{aligned}
$$

Here, $\left(\xi_{i}, \xi_{j}\right)$ is one of the chosen pairs and $\left(\xi_{i}^{\prime}, \xi_{j}^{\prime}\right)$ is the corresponding post-collisional pair. We set

$$
\tilde{\mu}_{\Delta t}^{n^{n}}=\sum_{i=1}^{n_{n}} g_{i}(\Delta t) \delta_{\xi_{\mathrm{i}}}+\sum_{i=1}^{n_{n}} g_{i}^{\prime}(\Delta t) \delta_{\xi_{i}^{\prime}}
$$

and ask whether

$$
\lim _{n \rightarrow \infty} E \mu_{\Delta t}^{n}=\mu_{\Delta}
$$


will hold. By repeating the first few steps in the proof of Theorem 3.4, we get

$$
\begin{aligned}
\left\langle\tilde{\mu}_{\Delta t}^{n}, \varphi\right\rangle= & \left\langle\mu_{0}, \varphi\right\rangle+\sum_{k=1}^{m} \Delta t(2 m)\left|S^{2}\right| \cdot q\left(\xi_{\pi(2 k-1)}, \xi_{\pi(2 k)}, e_{k}\right) \\
\times & \left\{\varphi\left(\xi_{\pi(2 k-1)}\right)+\varphi\left(\xi_{\pi(2 k)}\right)-\varphi\left(\xi_{\pi(2 k-1)}^{\prime}\right)-\varphi\left(\xi_{\pi(2 k)}^{\prime}\right)\right\} \\
& \times\left[g_{*(2 k-1)}^{\prime}(0) g_{*(2 k)}^{\prime}(0)-g_{\pi(2 k-1)}(0) g_{\pi(2 k)}(0)\right] .
\end{aligned}
$$

Let now $k=1, \ldots, 2 \mathrm{~m}$, and set

$$
\begin{aligned}
\xi_{\pi(2 k-1)}: & =\xi_{\pi((2 k-1)-2 m)}^{\prime} \\
\xi_{\pi(2 k)}: & =\xi_{\pi(2 k-2 m)}^{\prime}
\end{aligned}
$$

for $m+1 \leq k \leq 2 m$ (i.e. we "add" the post-collisional velocities to our velocity set). Equivalently,

$$
\begin{aligned}
\xi_{\pi(2 k)}^{\prime} & =\xi_{\pi(2 k+2 m)} \\
\xi_{\pi(2 k-1)}^{\prime} & =\xi_{\pi((2 k-1)+2 m)}
\end{aligned}
$$

for $k=1, \ldots, m$, and the collision term in (29) can be written in the symmetrized form

$$
\begin{gathered}
\sum_{k=1}^{2 m} \Delta t(2 m)\left|S^{2}\right| \cdot q\left(\xi_{\pi(2 k-1)} \xi_{\pi(2 k)}, e_{k}\right) \\
\times\left\{\varphi\left(\xi_{\pi(2 k-1)}^{\prime}\right)+\varphi\left(\xi_{\pi(2 k)}^{\prime}\right)-\varphi\left(\xi_{\pi(2 k-1)}\right)-\varphi\left(\xi_{\pi(2 k)}\right)\right\}\left[g_{\pi(2 k-1)}(0) g_{\pi(2 k)}(0)\right] .
\end{gathered}
$$

The problem which we face at this point is that even though (by construction) $\mu_{0}^{n} \rightarrow \mu_{0}$ in any of the meanings given by Lemma 3.1, we cannot prove anymore that the expectation of the above sum converges to

$$
\frac{1}{2} \Delta t \iiint_{S^{2}} q\left(\xi, \xi_{*}, e\right)\left\{\varphi^{\prime}+\varphi_{*}^{\prime}-\varphi-\varphi_{*}\right\} d \mu_{0}(\xi) d \mu_{0}\left(\xi_{*}\right) d e
$$

The difficulty is that while the collision pairs $\xi_{\pi(2 k-1)}, \xi_{\pi(2 k)}, k=1, \ldots, m$ were formed in a completely random and independent way, the collision pairs $\left(\xi_{\pi(2 k-1)}^{\prime}, \xi_{\pi(2 k)}\right)$, $k=1, \ldots, m$ are fixed once the random choices for the original velocities are made. This introduces correlations into the scheme which may destroy the convergence to the product measure. To illustrate the problem, we use the following simple example, where assume that $\mu_{0}=\lambda$ (the Lebesgue measure) on the unit interval $[0,1]$.

Suppose that we have a sequence of point sets $\left\{x_{1}, \ldots, x_{n}\right\} \subset[0,1]$, filling the interval such that $\frac{1}{n} \sum \delta_{x_{i}} \rightarrow \lambda$ weak $-*$. Moreover, assume that an operation like the collision transformation, applied to randomly chosen pairs $\left(x_{i}, x_{j}\right)$ from each set, yields "post-collisional" sets $x_{1}^{\prime}, \ldots, x_{n}^{\prime}$, which offer additional points to improve the approximation.

To make our argument transparent, let us assume that these "post-collisional" points are distributed even enough such that we can approximate $\lambda$ on certain subintervals of $[0,1]$ by using only post-collisional points. Specifically, suppose that 
we can approximate $\lambda$ by pre-collisional points on $\left[0, \frac{1}{2}\right)$ and by post-collisional points on $\left[\frac{1}{2}, 1\right]$. Such a choice would make the above convergence involving the product measure $\lambda \otimes \lambda$ impossible, because collision pairs could only be of the type $\left(x_{i}, x_{j}\right)$ or $\left(x_{i}^{\prime}, x_{j}^{\prime}\right)$, but never $\left(x_{i}, x_{j}^{\prime}\right)$. Because none of these pairs would lie in $\left(0, \frac{1}{2}\right) \times\left(\frac{1}{2}, 1\right)$ or $\left(\frac{1}{2}, 1\right) \times\left(0, \frac{1}{2}\right)$, the limit measure could not be equal to $\lambda \times \lambda$.

The distribution of the post-collisional velocities is clearly the big unknown in this argument (and, incidentally, also in any verification of our assumptions $\mathrm{Al}$ and A2 in Theorem 3.4). We plan to address this problem in future work.

Acknowledgment. The first named author (R.I.) would like to express his gratitude to the Institut of Mechanics at the University of Paris VI, where this research was started, to the Department of Mathematics at the University of Kaiserslautern, where it was pursued, and to the German Research Foundation (DFG), which helped to fund it. The research was also supported in part by the Natural Sciences and Engineering Research Council of Canada under grant Nr. 7847.

\section{References}

1. Broadwell J., 1964, Shock structure in a simple discrete velocity gas, Phys. Fluids 7, 1243-1247

2. Platkowski T., 1991, Boundary value problems for a class of the Broadwell models in bounded domains with general boundary data, Transport Th. Stat. Phys. $20(2 \& 3), 199-220$

3. DiPerna R., Lions P.L., 1989, On the Cauchy problem for the Boltzmann equation: Global existence and weak stability, Ann. Math. 130, 321-366

4. Bony J.M., 1991, Existence globale à données de Cauchy petites pour les modèles discrets de l'équation de Boltzmann, Commun. Partial Differential Equations

5. Cercignani C., Illner R., Shinbrot M., 1987, A boundary value problem for discrete-velocity models, Duke Math. I. 55 (4), 889-900

6. Cornille H., 1987, Exact solutions of the Broadwell equations in $1+1$ dimensions, J. Phys. A: Math. Gen. 20, 1973-1988

7. Cabannes H., Tiem D. H., 1987, Exact solutions for some discrete models of Boltzmann equation, Complex Systems 1, 575-585

8. Greenberg J., in Nonlinear Hyperbolic Waves, IMA 1989

9. Illner R., Platkowski T., 1988, Discrete velocity models of the Boltzmann equation: A survey on the mathematical aspects of the theory, SLAM Rew. 30 (2), $213-255$

10. Sultangazin U, Discrete nonlinear models of the Boltzmann equation. Transl. from the Russian Nauka Publ, Gen. Ed. Board Foreign Lang. Publ., Moscow 1987

11. Nurlybaev N.A., 1991, Discrete models of the Boltzmann equation, in: Abstracts of Euromech Colloquium 285. "Kinetic Theory, Transfer Processes of Evaporation and Condensation", pp. 39-41, Minsk 1991 


\section{Babovsky H., Il}

method for the full Bolt 13. Babovsky H., 19 scheme, Europ. J. Mech 14. Wagner W., 199 Carlo method for the $B$

*) permanent addres Box 3045, Victoria, B.C 